\title{
Effect of hepatic injury on adjuvant arthritis
}

\author{
ROBERT S. PINALS \\ From the Departments of Medicine and Rehabilitation Medicine, State University of New York, Upstate Medical \\ Center, Syracuse, New York
}

Hench (1933) described the ameliorating effect of hepatic disease on rheumatoid arthritis. Although jaundice was thought to be the determining factor in these remissions, it was not possible to reproduce the phenomenon by administration of bilirubin and bile salts (Hench, 1940), and a satisfactory explanation of its mechanism has never been offered (Hill and Holley, 1966). This was investigated in the present study using the rat adjuvant arthritis (AA) model. With the hepatotoxin dimethylnitrosamine (DMN), it is possible to produce a necrotizing hepatitis, which resolves rapidly, resulting in a virtually normal histological appearance in about 2 weeks (Barnes and Magee, 1954). Since DMN is converted by the action of liver enzymes to an alkylating intermediate which, in turn, is rapidly inactivated, it functions as a selective hepatotoxin (Magee and Swann, 1969). Thus a brief but severe episode of liver injury may be superimposed on AA at various points during its induction and course.

\section{Material and methods}

Male Carworth rats weighing 120 to $175 \mathrm{~g}$. were injected in the tail with $0 \cdot 1 \mathrm{ml}$. complete Freund's adjuvant, $\boldsymbol{M}$. butyricum 0.8 per cent. in light mineral oil. More than 95 per cent. of the animals developed joint swelling, which was usually first observed on the 10th day after injection, reached a peak 6 to 8 days after onset, and subsided gradually over a period of a month or more. The number of definitely swollen joints was recorded each day by a technician, without knowledge of the treatment group in which the individual animals were included. An index of peripheral joint swelling was derived as follows:

$$
\frac{\text { Number of swollen joints }}{\text { Total number of joints }} \times 10=\text { Arthritis Score }
$$

DMN was administered intraperitoneally in saline. Control animals received saline. Several experiments were performed, in which liver, obtained from both normal rats and from rats injected with DMN from 2 to 6 days previously, was homogenized and extracted with cold saline. Extracts were injected into recipient rats within 30 min. of killing the donors. In several experiments plasma for corticosterone determination (Mattingly, 1962) was obtained from cardiac blood between 9 and 10 a.m., within 3 minutes after intraperitoneal injection of pentobarbital.

\section{Results}

(1) EFFECT OF DMN IN THE NORMAL RAT

Rats developed a centrilobular necrosis within $48 \mathrm{hrs}$ of DMN injection (Fig. 1, overleaf). Regeneration was noted after 4 days, resulting in restoration of normal architecture within 10 to 14 days, except for mild fibrosis around the central vein. These changes were not produced by doses less than $15 \mathrm{mg} . / \mathrm{kg}$. and were inconsistent with doses between 15 and 20 $\mathrm{mg} . / \mathrm{kg}$. Necrosis invariably resulted with doses in the range of $20-30 \mathrm{mg}$. $/ \mathrm{kg}$., with a mortality of 3 per cent. after a single injection, rising to 24 per cent. if a second injection was given 4 days later, during the regeneration phase. After DMN injection there was slight weight loss or failure to gain for 2 days, after which weight increased at the pre-injection rate. No significant changes occurred in serum bilirubin in eight rats killed from 2 to 7 days after DMN injection, as compared with control rats. In twenty rats killed 1 or 2 days after DMN injection, mean plasma corticosterone values were slightly lower $(0.144 \mu \mathrm{g} . / \mathrm{ml}$.) than in eleven uninjected rats $(0 \cdot 153 \mu \mathrm{g} . / \mathrm{ml}$.).

(2) EFFECT OF DMN INJECTION ON THE DEVELOPMENT OF ARTHRITIS

A series of experiments was performed to detect inhibition of AA by injection of DMN $20-30 \mathrm{mg}$. $/ \mathrm{kg}$. at various times in relation to the induction and development of arthritis. A control group of rats receiving only adjuvant was included in each trial. No inhibition was demonstrated when DMN was given two days before adjuvant and on Days 2, 3, 6, 7, and 8 after adjuvant. DMN given on Day 20, i.e. 10 days after the development of arthritis, was also ineffectual. In four separate experiments, partial suppression of AA was demonstrated when a single dose of DMN 30 mg./kg. was given on Days 4 or 5 after adjuvant. Combined results of two such experiments in which DMN was given on Day 5 is shown in Fig. 2(overleaf). 


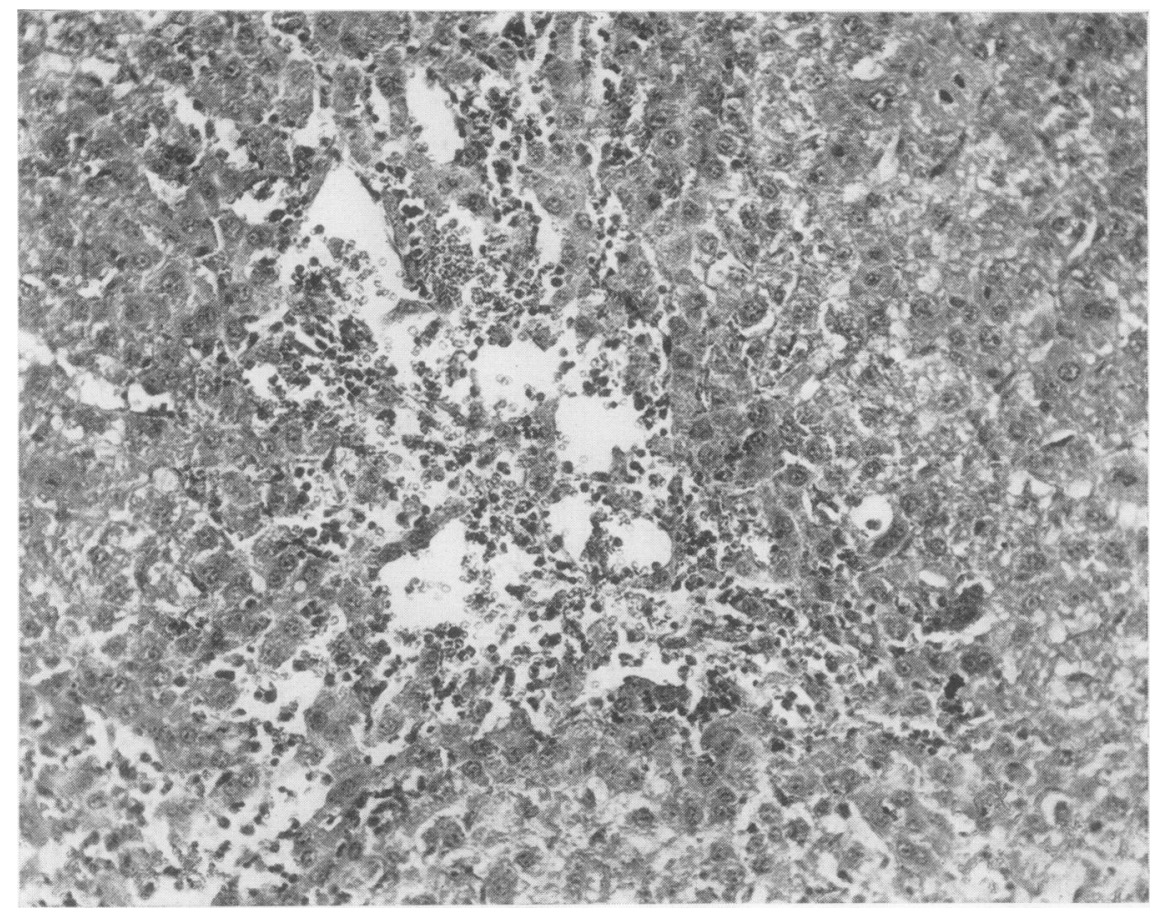

FIG. 1 Rat liver 72 hrs after administration of DMN,30 mg./kg., showing centrilobular necrosis with exudation of moder numbers of red and white blood cells. $\times 140$

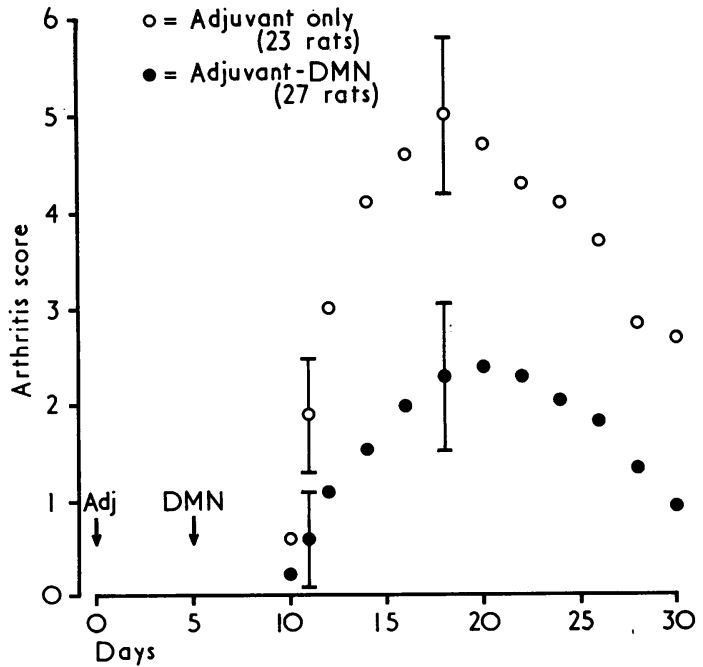

FIG. 2 Mean arthritis score $( \pm S E)$ lower in rats injected with DMN $30 \mathrm{mg} . / \mathrm{kg} .5$ days after adjuvant

Almost identical results were obtained with DMN injection on Day 4.

(3) EFFECT OF INJECTION OF LIVER EXTRACT ON THE DEVELOPMENT OF ARTHRITIS

In order to investigate the possibility that some material present in and released from necrotic liver

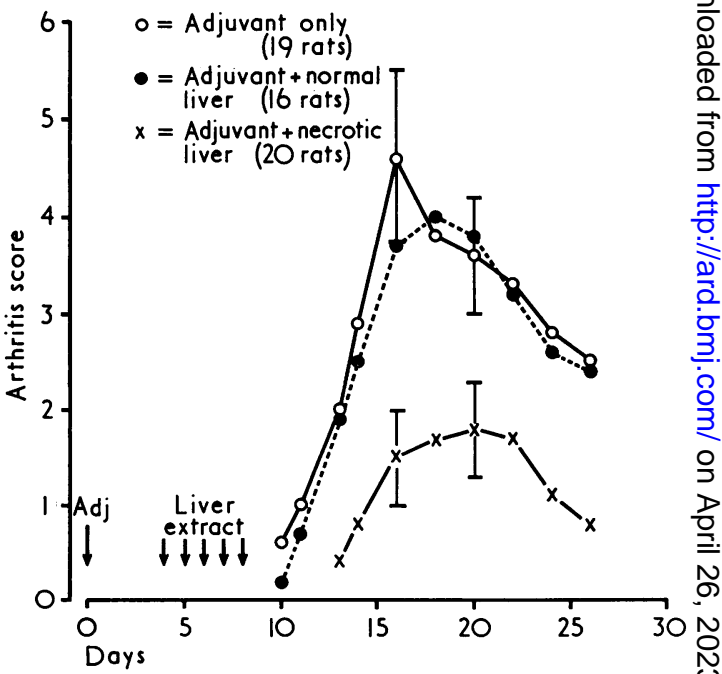

FIG. 3 Mean arthritis score $( \pm S E)$ lowest in rats injected with necrotic liver

might be responsible for suppression of AA, fourd experiments were performed, wherein saline extracts of liver were injected intraperitoneally. Rats were injected daily for 5 days, starting on the fourth day after adjuvant injection with the extract of $1 \mathrm{~g}$. livero (wet weight). Extracts were made of normal rat liver and of necrotic liver removed from 2 to 6 days after 
injection of DMN into the donor rats. Thus, the first injection consisted of extract from a liver removed 2 days after $D M N$ and the second of extract from a liver removed 3 days after DMN. Combined results are shown in Fig. 3. The use of normal liver extracts produced no alteration in the time of onset or the severity of AA, whereas injection of necrotic liver extracts delayed onset and diminished intensity of arthritis, to a degree comparable with that seen in previous experiments with DMN injection. No effect on intensity of arthritis was seen when half the amount of DMN-liver extract was used (equivalent to $0.5 \mathrm{~g}$. liver), but the onset and peak of AA was delayed for approximately 2 days

\section{Discussion}

Clinical observations of patients with rheumatoid arthritis have not led to an understanding of the remission induced by hepatocellular disease and jaundice. The phenomenon does not occur with haemolytic jaundice, nor can it be reproduced by administration of bilirubin (Hench, 1940). Although some cases have had biliary obstruction, most have had either toxic or virus hepatitis (Hench, 1940). Deliberate induction of virus hepatitis resulted in a temporary remission, with a mean duration of $\mathbf{4 0}$ days, in 25 of 32 patients with rheumatoid arthritis (Gardner, Stewart, and MacCallum, 1945). It is noteworthy that in several cases improvement in joint symptoms preceded the development of jaundice, an observation also made by Hench (1940). Although alteration of endogenous steroid metabolism has been presumed to be the mechanism of remission, this has never been demonstrated directly (Hill and Holley, 1966). The rate of cortisol conjugation may decrease in acute and chronic liver disease, but this results in diminished ACTH release and a steady state in which cortisol synthesis is reduced and plasma levels are normal (Peterson, 1960), although diurnal variation of plasma cortisol may be lost (Tucci, Albacete, and Martin, 1966).

In these experiments it was shown that a chemicallyinduced toxic hepatitis could partially inhibit the development of AA. Jaundice did not develop, as was anticipated from other studies in which rats given hepatotoxins demonstrated a remarkable capacity to handle large exogenous bilirubin loads (Krueger and Higginson, 1961). The timing of liver necrosis was critical, for no inhibition resulted when the toxin was administered too soon, i.e. before adjuvant or within 3 days afterwards, or too late, i.e. within 4 days before the development of overt signs of arthritis. Since various treatments which are thought to inhibit AA through immunosuppression, such as antilymphocyte globulin (Currey and Ziff, 1968) and cyclophosphamide (Brown, Taylor and Pollock, 1971), are most effective when administered before or soon after adjuvant injection, the response to hepatic necrosis should probably be regarded as an anti-inflammatory effect. The inhibition could be reproduced by injection, at the appropriate time, of necrotic liver extracts, but not by injection of normal liver extracts. This would support the possibility that some anti-inflammatory material released from necrotic liver was responsible for inhibition of AA.

Several alternative explanations may be offered:

(1) DMN might have a direct anti-inflammatory effect Suppression of AA by a single dose of any drug given well after induction of the disease by adjuvant would not conform to broad experience with other agents in this system. Our understanding of DMN metabolism would indicate that it should not be present in active form in liver extracts obtained 2 days after administration (Magee and Swann, 1969), thus failing to account for inhibition of AA by these extracts.

(2) Endogenous corticosteroid release provoked by the stress of hepatic necrosis, or failure of the damaged liver to metabolize corticosteroids could be responsible for the inhibition of $A A$

The failure to find elevation of plasma corticosterone levels in DMN-injected rats provides some evidence against this mechanism, but definitive experiments using adrenalectomized rats were not attempted because of the high mortality rate after adjuvant injection reported by others (Glenn and Gray, 1965).

(3) Endotoxin might be the mediator of AA suppression Animals with severe liver injury are more susceptible to the effects of endotoxin (Farrar and Magnani, 1964). Although endotoxin may inhibit AA, it does so most effectively when administered before adjuvant injection (Wood and Pearson, 1962).

The role of the liver in the suppression of inflammation has been investigated recently by Billingham, Gordon, and Robinson (1971). These workers have shown that the liver synthesizes an anti-inflammatory protein which is capable of inhibiting oedema formation, granulation tissue deposition, and delayed hypersensitivity, all of which may play a role in AA. Mielens, Drobeck, Rozitis, and Sansone (1969) have demonstrated inhibition of cotton granuloma and carrageenin oedema in intact and adrenalectomized rats with oral administration of carbon tetrachloride. However, Walz, DiMartino, and Misher (1971) found no modification of AA with the use of this hepatotoxin. McArthur, Dawkins, Smith, and Hamilton (1971) have proposed that certain circulating peptides possess anti-inflammatory properties and that their displacement from serum binding proteins may be an important mode of action of various anti-inflammatory drugs. They measured free and bound serum tryptophan, which, they suggested, may mimic the behavior of anti-inflammatory peptides, and found 
that free tryptophan was increased in patients with jaundice and pregnancy as well as in rheumatoid arthritis under treatment with various medications.

Although these observations fall far short of an explanation for the remissions induced by liver disease in rheumatoid arthritis, they suggest that release of an anti-inflammatory material or activator may be a mechanism worthy of further investigation.

\section{Summary}

Adjuvant arthritis was partially inhibited by the administration of a hepatotoxin, dimethylnitrosamine, on the fourth or fifth day after adjuvant injection. Injection of necrotic liver extracts, but not normar? liver extracts, also resulted in a similar suppression of arthritis. The experiments supported the possibility that release of some anti-inflammatory material frome damaged liver might be the mechanism of remission in rheumatoid arthritis patients who develop hepatocellular disease.

The advice of Dr. Byron Hamilton and the technical assistance of Friedel Ehrlinspiel were appreciated. The study was supported by a Clinical Research Center Grant from the Arthritis Foundation. Corticosterone determ- inations were kindly performed by Dr. David Streeten.

\section{References}

BARnes, J. M., AND MaGee, P. N. (1954) Brit. J. industr. Med., 11, 167 (Some toxic properties of dimethylnitrosamine)

Billingham, M. E. J., Gordon, A. H., AND Robinson, B. V. (1971) Nature (New Biology), 231, 26 (Role of the liver in? inflammation)

Brown, J. H., TaYlor, J. L., AND Pollock, S. H. (1971) Arch. intern. Pharmacodyn., 194, 381 (Effect of duration of administration of cyclophosphamide and steroidal anti-inflammatory drugs on the severity of polyarthritis)

CURReY, H. L. F., AND ZIFF, M. (1968) J. exp. Med., 127, 185 (Suppression of adjuvant disease in the rat by heterologous antilymphocyte globulin)

FarRar, W. E., JR., AND MAGNANI, T. J. (1964) Proc. Soc. exp. Biol. (N.Y.), 115, 596 (Endotoxin susceptibility following hepatic injury by carbon tetrachloride)

Gardner, F., StewarT, A., AND MacCallum, F. O. (1945) Brit. med.J., 2, 677 (Therapeutic effect of induced jaundice in rheumatoid arthritis)

GlenN, E. M., AND Gray, J. (1965) Amer.J. Vet. Res., 26, 1180 (Adjuvant-induced polyarthritis in rats: Biologic and histologic background)

Hench, P. S. (1933) Proc. Mayo Clin., 8, 430 (Analgesia accompanying hepatitis and jaundice in cases of chronic arthritis, fibrositis, and sciatic pain)

- (1940) Med. Clin. N. Amer., 24, 1209 (The advantages of hepatic injury and jaundice in certain conditions, notably the rheumatic diseases)

HiLl, S. R., JR, AND HolLEY, H. L. (1966) 'The ameliorating effect of pregnancy and jaundice on certain rheumatic diseases,' in 'Arthritis and Allied Disorders', 7th ed., ed. J. L. Hollander, p. 612. Lea and Febiger, Philadelphia.

Krueger, H., AND Higginson, J. (1961) Proc. Soc. exp. Biol. (N.Y.), 107, 43 (The bilirubin overload test with special reference to liver damage produced by India ink)

Magee, P. N., AND SwanN, P. F. (1969) Brit. med. Bull., 25, 240 (Nitroso compounds)

Mattingly, D. (1962) J. clin. Path., 15, 374 (A simple fluorometric method for the estimation of free 11hydroxycorticoids in human plasma)

McArthur, J. N., Dawkins, P. D., Smith, M. J. H., AND Hamilton, E. B. D. (1971) Brit. med. J., 2, 677 (Mode of action of antirheumatic drugs)

Mielens, Z. E., Drobeck, H. P., Rozitis, J., JR, ANd Sansone, V. J., JR (1969) Toxicol. appl. Pharmacol., 14, 293 (Inhibition of experimental inflammation by oral toxic agents)

Peterson, R. S. (1960) J. clin. Invest., 39, 320 (Adrenal cortical steroid metabolism and adrenocortical function in liver disease)

Tucci, J. R., Albacete, R. S., AND Martin, M. M. (1966) Gastroenterology, 50, 637 (Effect of liver disease upon steroid circadian rhythms in man)

Walz, D. T., DiMartino, M. J., AND Misher, A. (1971) J. Pharm. exp. Ther., 178, 223 (Adjuvant-induced arthritis in rats. II. Drug effects on physiologic, biochemical and immunologic parameters)

Wood, F. D., AND Pearson, C. M. (1962) Science, 137, 544 (Protection of rats against adjuvant arthritis by bacterial lipopolysaccharides) 